\title{
Two Composite Higgs Doublets: Is it the Low Energy Limit of a Natural Strong Electroweak Symmetry Breaking Sector?
}

\author{
Alfonso R. Zerwekh* \\ Centro de Estudios Subatómicos and Instituto de Física, \\ Facultad de Ciencias, \\ Universidad Austral de Chile, \\ Casilla 567, Valdivia,Chile
}

\begin{abstract}
In this paper, we propose an effective model scheme that describes the electroweak symmetry breaking sector by means of composite Higgslike scalars, following the ideas of Minimal Walking Technicolor (MWT). We argue that, because of the general failure of Extended Technicolor (ETC) to explain the mass of the top quark, it is necessary to introduce two composite Higgs bosons: one of them originated by a MWT-ETC sector and the other one produced by a Topcolor sector. We focus on the phenomenological differences between the light composite Higgs present in our model and the fundamental Higgs boson predicted by the Standard Model and their production at the LHC. We show that in this scheme the main production channel of the lighter Higgs boson are the associated production with a gauge boson and $W W$ fusion but not the gluon-gluon fusion channel which is substantially suppressed.
\end{abstract}

\section{Introduction}

High Energy Physics is at the dawn of a new era. In the very near future the LHC will allow us to explore, for the first time, the physics responsible for the electroweak symmetry breaking. We are quite sure that something different to the minimal Higgs sector of the Standard Model will be found, but the conjectured alternatives, far from being unique, are varied and very different among them, going from Higgsless models to Supersymmetry.

A still very appealing possibility is the existence of a new strong interaction at the $\mathrm{TeV}$ scale that breaks the electroweak symmetry by the formation of condensates of (techni-)fermions. This kind of models receives the generic

*alfonsozerwekh@uach.cl 
name of Technicolor (TC) [1. Nevertheless, the idea of a new strong dynamics responsible for the mass of the gauge bosons has been seriously challenged by the precision measurements made at LEP. The main source for the discrepancy was the underlying and unnecessary hypothesis that Technicolor was essentially a scaled up version of QCD. Nevertheless, during the last few years, new models of Technicolor have been built exhibiting interesting properties like: minimal number of technifermions leading to acceptable values of the $S$ parameter, quasi-conformal behavior of the coupling constant ("walking" Technicolor) and the presence, in the low energy spectrum, of a light Higgs-like composite scalar 2, 3, 4, 5, 6, 7. Phenomenological studies have been done, showing that such a composite Higgs boson and the composite vector bosons predicted by the models are able to produce interesting signals at the LHC such as the enhancement, with respect to the Standard Model, of the associated production of a Higgs and a gauge boson 8,9 .

Despite the success of these new ideas, a fundamental problem remains in such a dynamical approach to the electroweak physics: the origin of the fermion masses. A traditional solution has been to extend the Technicolor interaction to include standard fermions. This idea works very well for the two lighter generations, but is unable to explain the huge mass of the top quark. A viable solution is the so called Topcolor Assisted Technicolor [10 where a new strong interaction, Topcolor (TopC) generates a dynamical mass for the top quark while Technicolor provides mass to the $W^{ \pm}$and $Z^{0}$ gauge bosons and the masses of the fermions belonging to the first two families are generated by Extended Technicolor (ETC) .

In this paper, we take seriously these ideas and we propose that the low energy limit of an underlying Topcolor Assisted Technicolor theory must contain in its spectrum two composite Higgs doublets: one for generating the masses of the the $W^{ \pm}$and $Z^{0}$ gauge bosons and the masses of the light fermions and another for generating the masses of the fermions of the third generation. We emphasize that the phenomenology of the resulting light Higgs boson is necessarily very different from the standard expectations.

As an example, we construct an effective low energy Lagrangian that combine the main features of (Minimal) Walking Technicolor and Topcolor Assisted Technicolor. We assume, for example, that MWT is able to produce a light composite Higgs boson that, from the effective theory point of view, is able to break the electroweak symmetry and gives mass (via Yukawa coupling that have their origin in ETC) to the first two families of fermions. On the other hand, we postulate the existence of an additional and maybe heavier composite Higgs boson coming from a Topcolor sector that only couples to the third family. We include also some vector resonances for each strong sector.

\section{The Model}

We start by generalizing the phenomenological model introduced in reference 8. In the present description we are going to include, beside the fields of the 
Standard Model (which we call $V_{0}$ and $B_{0}$ ), two isotriplet spin 1 fields (which we call $V_{1}$ and $V_{2}$ ), two isosinglet spin 1 fields $\left(B_{1}, B_{2}\right)$ and two complex scalar doublets: $\varphi$ and $\Phi$. We assume that $V_{1}, B_{1}$ and $\varphi$ are composite fields produced by Technicolor and couple to the first and second fermion generations, while $V_{2}, B_{2}$ and $\Phi$ are Topcolor bound states and couple to the third generation. If we work in a basis where $V_{0}$ (the elementary "proto"- $W$ vector field), $V_{1}$ and $V_{2}$ transform as gauge fields under $S U(2)_{L}$ while $B_{0}, B_{1}$ and $B_{2}$ transform as gauge fields of $U(1)$; the Lagrangian of the gauge sector can be written as:

$$
\begin{aligned}
\mathcal{L}_{\text {gauge }}= & -\frac{1}{4} V_{0 \mu \nu}^{a} V_{0}^{a \mu \nu}-\frac{1}{4} V_{1 \mu \nu}^{a} V_{1}^{a \mu \nu}-\frac{1}{4} V_{2 \mu \nu}^{a} V_{2}^{a \mu \nu} \\
& +\frac{M_{1}^{2}}{2 g_{1}^{2}}\left(g_{0} V_{0}-g_{1} V_{1}\right)^{2}+\frac{M_{2}^{2}}{2 g_{2}^{2}}\left(g_{1} V_{1}-g_{2} V_{2}\right)^{2} \\
& -\frac{1}{4} B_{0 \mu \nu} B_{0}^{\mu \nu}-\frac{1}{4} B_{1 \mu \nu} B_{1}^{\mu \nu}+\frac{M_{3}^{2}}{2 g_{1}^{\prime 2}}\left(g_{0}^{\prime} B_{0}-g_{1}^{\prime} B_{1}\right)^{2} \\
& +\frac{M_{4}^{2}}{2 g_{2}^{\prime 2}}\left(g_{1}^{\prime} B_{1}-g_{2}^{\prime} B_{2}\right)^{2}
\end{aligned}
$$

where $g_{i}\left(g_{i}^{\prime}\right)$ are the coupling constants associated to the vector bosons $V_{i}\left(B_{i}\right)$ (with $i=0,1,2)$.

At this point, we want to point out that it would be straightforward to introduce axial-vector fields which would be necessary to minimize the value of the $S$ parameter at the phenomenological level, nevertheless we include only vector resonances in order to focus on the main features of the model, specially those related to the composite Higgs.

For the Higgs sector, we use a CP-conserving two doublets Higgs Lagrangian [1]:

$$
\begin{aligned}
\mathcal{L}_{\text {Higgs }}= & \left(D_{\mu} \varphi\right)^{\dagger}\left(D^{\mu} \varphi\right)+\left(D_{\mu} \Phi\right)^{\dagger}\left(D^{\mu} \Phi\right) \\
& -\mu_{1}^{2}\left(\varphi^{\dagger} \varphi\right)-\mu_{2}^{2}\left(\Phi^{\dagger} \Phi\right)+\left[\mu_{12}^{2}\left(\Phi^{\dagger} \varphi\right)+\text { h.c. }\right] \\
& -\frac{\lambda_{1}}{2}\left(\varphi^{\dagger} \varphi\right)^{2}-\frac{\lambda_{2}}{2}\left(\Phi^{\dagger} \Phi\right)^{2}-\lambda_{3}\left(\Phi^{\dagger} \Phi\right)\left(\varphi^{\dagger} \varphi\right) \\
& -\lambda_{4}\left(\Phi^{\dagger} \varphi\right)\left(\varphi^{\dagger} \Phi\right)-\left[\frac{1}{2} \lambda_{5}\left(\Phi^{\dagger} \varphi\right)^{2}+\text { h.c. }\right]
\end{aligned}
$$

where

$$
\begin{aligned}
D_{\mu} \varphi= & \partial_{\mu} \varphi+i \tau^{a} g_{0}(1-f) V_{0 \mu}^{a} \varphi \\
& +i \tau^{a} g_{1} f V_{1 \mu}^{a} \varphi+i(1-k) \frac{Y}{2} g_{0}^{\prime} B_{0 \mu} \varphi \\
& +i k \frac{Y}{2} g_{1}^{\prime} B_{1 \mu} \varphi
\end{aligned}
$$




$$
D_{\mu} \Phi=\left(\partial_{\mu}+i \tau^{a} g_{2} V_{2 \mu}^{a}+i \frac{Y}{2} g_{2}^{\prime} B_{2 \mu}\right) \Phi
$$

and $f$ and $k$ are phenomenological parameters that assure gauge invariance.

We want now to emphasize an important aspect of our model. Because we are working in a basis where $V_{0}, V_{1}$ and $V_{2}$ (all of them) transform as gauge fields of the same group $S U(2)_{L}$, any linear combination $\alpha_{0} g_{0} V_{0}+\alpha_{1} g_{1} V_{1}+\alpha_{2} g_{2} V_{2}$ ( where $\alpha_{0}, \alpha_{1}$ and $\alpha_{2}$ are some parameters) transforms like a gauge field and can be used to construct a covariant derivative, provided that $\alpha_{0}+\alpha_{1}+\alpha_{2}=1$. Evidently, a similar feature happens in the $U(1)_{Y}$ sector. This property allows us to couple $\Phi$ only with $V_{2}$ and $B_{2}$ as in equation (4) and, therefore,suppress the contribution of $\Phi$ to the mass of the $W$ and $Z$ bosons because any contribution would come only from a mixing term (assumed to be small). On the other hand, in order to be consistent with the known phenomenology, we can always include in the covariant derivative, in the Dirac term of the third generation quarks, the general linear combination of vector fields in order to guarantee that the top quark couples to the $W$ and $Z$ bosons in the usual way. More details about this method of treating vector resonances can be found in [12 and 8 .

We assume that both scalar doublets get a vacuum expectation value:

$$
\langle\varphi\rangle=\frac{1}{\sqrt{2}}\left(\begin{array}{c}
0 \\
v_{1}
\end{array}\right),\langle\Phi\rangle=\frac{1}{\sqrt{2}}\left(\begin{array}{c}
0 \\
v_{2}
\end{array}\right) .
$$

In order to simplify our model, focusing on the main physical ideas, we are going to work in the limit where $g_{2}$ and $g_{2}^{\prime}$ are large enough to produce an effective decoupling of $V_{2 \mu}^{a}$ and $B_{2 \mu}$ from the rest of vector fields in (1). In this limit we re-obtain the model described in 8 for the TC-ETC sector plus an additional Topcolor sector. When the Higgs doublets get their vacuum expectation values, the photon and the $W^{ \pm}$and $Z$ bosons can be written in terms of the original fields as:

$$
\begin{aligned}
A= & \frac{g_{0}^{\prime}}{\sqrt{g_{0}^{2}+g_{0}^{\prime 2}}} V_{0}^{3}+\frac{g_{0}}{\sqrt{g_{0}^{2}+g_{0}^{\prime 2}}} B_{0}+\frac{g_{0} g_{0}^{\prime}}{g_{1} \sqrt{g_{0}^{2}+g_{0}^{\prime 2}}} V_{1}^{3} \\
& +\frac{g_{0} g_{0}^{\prime}}{g_{1} \sqrt{g_{0}^{2}+g_{0}^{\prime 2}}} B_{1} \\
Z= & \frac{g_{0}}{\sqrt{g_{0}^{2}+g_{0}^{\prime 2}}} V_{0}^{3}-\frac{g_{0}^{\prime}}{\sqrt{g_{0}^{2}+g_{0}^{\prime 2}}} B_{0}+\frac{g_{0}^{2}}{g_{1} \sqrt{g_{0}^{2}+g_{0}^{2}}} V_{1}^{3} \\
& -\frac{g_{0}^{\prime 2}}{g_{1} \sqrt{g_{0}^{2}+g_{0}^{\prime 2}}} B_{1} \\
W^{ \pm}= & \frac{1}{\sqrt{2}}\left\{\left(V_{0}^{1} \mp i V_{0}^{2}\right)+\frac{g_{0}}{g_{1}}\left(V_{1}^{1} \mp i V_{1}^{2}\right)\right\}
\end{aligned}
$$

From (3) and (4) we see that $\varphi$ couples to $V_{0}, V_{1}, B_{0}$ and $B_{1}$ while $\Phi$ only couples to the Topcolor generated vector resonances $\left(V_{2}\right.$ and $\left.B_{2}\right)$. As a 
consequence, only $\varphi$ generates the mass of the $W^{ \pm}$. This fact obligates us to identify $v_{1}$ with the electroweak scale $246 \mathrm{GeV}$ while $v_{2}$ remains unconstrained. Nevertheless, we still can suppose that $v_{2}$ can be of the order of the weak scale and, hence, $v_{2} \sim v_{1}$.

We will use the vacuum expectation value of $\Phi$ for producing the mass of the third generation fermions through an usual Yukawa interaction. Moreover, $\Phi$ generates (additional) mass for the TopC vector resonances. As is usual in models with two Higgs doublets, after the electroweak symmetry breaking and diagonalizing the mass matrix of the Higgs sector, charged Higgs bosons $\left(H^{ \pm}\right)$ and a CP-odd scalar $\left(A^{0}\right)$ will appear in the physical spectrum. In our scheme, they have their origin in $\Phi$ and they correspond to the so called "top-pion" in Topcolor Assisted Technicolor models. Therefore, they will couple strongly to the third generation. On the other hand, we expect that the lighter mass eigenstate, which will be mainly Technicolor-generated, will couple to the third family fermions with a reduced strength (compared to the SM).

Let's continue with the study of the Higgs sector. Lagrangian (2) has many free parameters and we need to make some simplifying assumptions. We chose to set up the following relations: $\mu_{12}=0, \lambda_{4}=\lambda_{5}$ and $\lambda_{5}<0$. This set of conditions keeps our expressions simple and still leads to an acceptable phenomenology. For example, $\mu_{12}=0$ is well motivated because it helps to restrict possible FCNC processes at tree and one loop level [11. With this choice, the minimum of the potential is obtained for:

$$
\begin{aligned}
\mu_{1}^{2} & =-\frac{1}{2} \lambda_{1} v_{1}^{2}-\frac{1}{2} \lambda_{3} v_{2}^{2} \\
\mu_{2}^{2} & =-\frac{1}{2} \lambda_{2} v_{2}^{2}-\frac{1}{2} \lambda_{3} v_{1}^{2}
\end{aligned}
$$

Of course, in order to have a true minimum we need that $\lambda_{1}>0, \lambda_{2}>0$ and $\lambda_{3}>-\sqrt{\lambda_{1} \lambda_{2}}$.

The masses of the charged Higgs and the CP-odd scalar are:

$$
m_{H^{ \pm}}^{2}=m_{A^{0}}^{2}=-v^{2} \lambda_{5}
$$

where $v^{2}=v_{1}^{2}+v_{2}^{2}$. We want to emphasize again that $v_{2}$ is not fixed by the mass of the $W$ and, in principle, can be large enough to maintain $m_{H^{ \pm}}^{2}$ and $m_{A^{0}}^{2}$ above their experimental limit while keeping $\lambda_{5}$ relatively small. In fact, because $\lambda_{5}$ and $\lambda_{3}$ are produced by the same underlying dynamics, it is not unreasonable to expect that $\left|\lambda_{5}\right|$ and $\left|\lambda_{3}\right|$ must be of the same order of magnitude.

In general, it is expected that top-pion's mass is of the order $200-400 \mathrm{GeV}$ 11. In our case, we can apply the limits on $m_{H^{ \pm}}$that have been obtained for the Two Higgs Doublet Model type III. In this context, it has been recently shown that FCNC restrictions imply that $m_{H^{ \pm}}>300 \mathrm{GeV}[13$.

Let's denote by $\tilde{h}$ and $\tilde{H}$ the radial excitations of $\varphi$ and $\Phi$ respectively around the new vacuum. The Higgs mass matrix in the basis $(\tilde{h}, \tilde{H})$ is: 


$$
\mathcal{M}^{2}=\left(\begin{array}{cc}
\lambda_{1} v_{1}^{2} & \left(\lambda_{3}+2 \lambda_{5}\right) v_{1} v_{2} \\
\left(\lambda_{3}+2 \lambda_{5}\right) v_{1} v_{2} & \lambda_{2} v_{2}^{2}
\end{array}\right)
$$

In the limit where $\lambda_{3}+2 \lambda_{5}$ is small, we can write the eigenvectors as:

$$
\begin{aligned}
h & =\tilde{h}-\theta \tilde{H} \\
H & =\tilde{H}+\theta \tilde{h}
\end{aligned}
$$

where $\theta$ is the mixing angle and is given by the following expression:

$$
\theta=\frac{v_{1} v_{2}\left(\lambda_{3}+2 \lambda_{5}\right)}{\left(m_{H}^{2}-m_{h}^{2}\right)}
$$

In the expression above, $m_{h}$ and $m_{H}$ are the masses of $h$ and $H$ respectively and, at this level of approximation, they are given by:

$$
\begin{aligned}
m_{h}^{2} & =\frac{1}{2} \lambda_{1} v_{1}^{2} \\
m_{H}^{2} & =\frac{1}{2} \lambda_{2} v_{2}^{2}
\end{aligned}
$$

At this point, it is worth to be remembered that $h$ is a mainly TC generated particle while $H$ is mainly a Topcolor composite state and we have assume from the beginning that $H$ is considerable heavier than $h$.

\section{Results}

One of the main consequences of the picture described above is that the light Higgs boson $h$ couples weakly to the top quark. By hypotheses, only $\tilde{H}$ couples to the third generation of fermions and, hence, $h$ gets its coupling to the top by the mixing term. Consequently, the coupling is proportional to $\theta$ which is assumed to be small. This means, also, that the effective $h$-gluon-gluon interaction (which is responsible for the main production mechanism of the standard Higgs at the LHC) will be significantly suppressed. Additionally, the decay of $h$ to $b \bar{b}$ will be also suppressed by the same reasons.

On the other hand, $h$ couples in the standard way to the gauge bosons $W^{ \pm}$ and $Z$; but also couples to Technicolor vector resonances. This fact implies that its associated production with gauge bosons will be enhanced as was found in 8 and 9 .

By contrast, $H$ couples in the normal way to the top quark. Additionally, in our simple model where we have completely decoupled the Topcolor generated vector resonances $V_{2}$ and $B_{2}$ from the other vector bosons, $\Phi$ (and hence $\tilde{H}$ ) doesn't couple to the gauge bosons $W^{ \pm}$and $Z$ (see equation (44). That means that $H$ couples weakly to the $W^{ \pm}$and $Z$, through it $\tilde{h}$ component. The strength of this interaction will be proportional to the small mixing angle $\theta$. 


\section{Conclusions}

In this work, we have proposed a scenario with two composite Higgs doublets that can be a viable low energy limit of an underlying complete and natural strong electroweak symmetry breaking sector which aims to explain not only the mass of the gauge bosons, but also the mass of the fermions. As a proof of concept, we constructed a model which implements this idea. It includes a Technicolor generated Higgs doublet which breaks the electroweak symmetry and gives mass to the first two generation of fermions, while a second, Topcolor generated, Higgs doublet gives mass only to the third generation fermions. An important ingredient of the model is the inclusion of vector resonances which allows us to implement the role separation between the two Higgs doublets. The different roles played by the two Higgs doublets implies that the phenomenology of the light composite Higgs boson is expected to be very different from that of the standard Higgs. In particular, we expect that its main production mechanisms at the LHC were the associated production with gauge bosons and $W W$ fusion, but not gluon fusion as happens in the Standard Model.

\section{Acknowledgments}

I want to thank to Rogrio Rosenfeld and Claudio Dib for very interesting discussions in the early stages of this work. I am also in debt with Maximiliano Rivera for the useful discussions about the potential of a two Higgs doublet model. The author is partially supported by Fondecyt grant 1070880 and by the Center of Subatomic Studies. TGD.

\section{References}

[1] C. T. Hill and E. H. Simmons, "Strong dynamics and electroweak symmetry breaking," Phys. Rept. 381 (2003) 235 [Erratum-ibid. 390 (2004) 553] arXiv:hep-ph/0203079.

[2] D. D. Dietrich, F. Sannino and K. Tuominen, "Light composite Higgs from higher representations versus electroweak precision measurements: Predictions for LHC," Phys. Rev. D 72 (2005) 055001 arXiv:hep-ph/0505059.

[3] D. D. Dietrich, F. Sannino and K. Tuominen, "Light composite Higgs and precision electroweak measurements on the $\mathrm{Z}$ resonance: An update," Phys. Rev. D 73 (2006) 037701 arXiv:hep-ph/0510217.

[4] F. Sannino, "Light composite Higgs: LCH @ LHC," Int. J. Mod. Phys. A 20 (2005) 6133 arXiv:hep-ph/0506205.

[5] S. B. Gudnason, C. Kouvaris and F. Sannino, "Towards working technicolor: Effective theories and dark matter," Phys. Rev. D 73 (2006) 115003 arXiv:hep-ph/0603014. 
[6] R. Foadi, M. T. Frandsen, T. A. Ryttov and F. Sannino, "Minimal Walking Technicolor: Set Up for Collider Physics," Phys. Rev. D 76 (2007) 055005 arXiv:0706.1696 [hep-ph]].

[7] T. A. Ryttov and F. Sannino, "Ultra Minimal Technicolor and its Dark Matter TIMP," Phys. Rev. D 78 (2008) 115010 [arXiv:0809.0713 [hep-ph]].

[8] A. R. Zerwekh, "Associate Higgs and gauge boson production at hadron colliders in a model with vector resonances," Eur. Phys. J. C 46 (2006) 791 arXiv:hep-ph/0512261.

[9] A. Belyaev, R. Foadi, M. T. Frandsen, M. Jarvinen, F. Sannino and A. Pukhov, "Technicolor Walks at the LHC," Phys. Rev. D 79 (2009) 035006 [arXiv:0809.0793 [hep-ph]].

[10] C. T. Hill, "Topcolor assisted technicolor," Phys. Lett. B 345 (1995) 483 arXiv:hep-ph/9411426.

[11] J. F. Gunion and H. E. Haber, "The CP-conserving two-Higgs-doublet model: The approach to the decoupling limit," Phys. Rev. D 67 (2003) 075019 arXiv:hep-ph/0207010].

[12] A. R. Zerwekh, "Effective description of a gauge field and a tower of massive vector resonances," arXiv:hep-ph/0307130.

[13] F. Mahmoudi and O. Stal, "Flavor constraints on the two-Higgs-doublet model with general Yukawa couplings," arXiv:0907.1791 [hep-ph]. 